\title{
Implementation Science to Improve Tobacco Cessation Services in Oncology Care
}

\author{
The Cancer Center Cessation Initiative Implementation Science Working Group*
}

\begin{abstract}
Every patient with cancer deserves access to evidence-based tobacco cessation interventions as part of their routine oncology care. The $\mathrm{NCl}$ Cancer Moonshot funded the Cancer Center Cessation Initiative (C3I) to help establish and/or expand tobacco treatment programs at $52 \mathrm{NCl}$-designated Cancer Centers. Although this initiative has broadened the availability of tobacco treatment services across US cancer centers, the reach and utilization of these services remains low among patients. To help address the remaining gap between the availability and utilization of evidence-based treatments for tobacco use in the oncologic context, staff and investigators at C3I sites and the C3I Coordinating Center formed the C3I Implementation Science Working Group. The mission of this working group is to bring together clinicians, scientists, and policymakers who share a common interest in implementation science and treating tobacco use in the oncologic context to collaborate on projects aimed at shrinking the practice gap in this area. Through case study examples, we describe how the C3I Implementation Science Working Group is supporting efforts to identify effective ways to increase the utilization of evidence-based tobacco treatments within cancer treatment settings and promote the broader impact and long-term sustainability of C3I.
\end{abstract}

J Natl Compr Canc Netw 2021;19(Suppl 1):S12-15 doi: 10.6004/jnccn.2021.7094

\section{Background}

Recognizing the persistent gap between the availability and use of evidence-based treatments to prevent and control cancer, ${ }^{1}$ a critical component of then Vice President Biden's Cancer Moonshot initiative was support for the NCl's commitment to implementation science, defined as the systematic study of methods to optimize the utilization of evidence-based treatments to improve health. Emerging from this commitment, the Cancer Center Cessation Initiative (C3I) was established to guide national efforts to increase the use of evidence-based tobacco cessation treatments within NCI-designated Cancer Centers. ${ }^{2}$ Despite the Surgeon General's Report establishing the causal link between tobacco use and poor clinical outcomes among patients with cancer and widespread availability of safe and effective tobacco treatments, ${ }^{3}$ only one-third of oncologists provide tobacco treatments to patients ${ }^{4}$ and only $15 \%$ of patients with cancer who continue to smoke report the use of cessation treatments. ${ }^{5}$ Support from NCI's C3I has expanded the use of evidence-based treatments for tobacco use across cancer centers, but challenges remain for ensuring acceptable reach, utilization, and sustainability. ${ }^{6}$

\footnotetext{
*A complete list of the collaborators in the Cancer Center Cessation Initiative Implementation Science Working Group appears at the end of this article.
}

To address remaining challenges, members of the C3I community, including staff and investigators at US cancer centers and the C3I Coordinating Center, formed the C3I Implementation Science Working Group. This working group's mission is to bring together clinicians, scientists, and policymakers who share a common interest in implementation science and treating tobacco use in the oncologic context. Leveraging national expertise in implementation science and tobacco research and NCI investments, including the NCI-funded Implementation Science Centers in Cancer Control (ISC3; https:// cancercontrol.cancer.gov/is/initiatives/isc3), the working group will identify effective implementation strategies to reduce the tobacco treatment practice gap within cancer care delivery (the specific working group agenda is described later), broadening the impact and sustainability (ie, the continuation of tobacco use treatment programs at sites after funding ends) of C3I. Here, we describe case studies of implementation science research within C3I and discuss the working group mission and goals.

\section{Case Studies of C3I Implementation Science Research}

C3I has supported 3 cohorts, totaling 52 NCI-designated Cancer Centers. Early implementation outcomes across sites, such as reach (ie, the proportion of patients assessed for tobacco use and engaged in evidence-based treatment) 
and effectiveness (ie, the proportion of patients who quit smoking), have been reported ${ }^{6-8}$ and learnings from initial cohorts were passed on to later cohorts via webinars and site-to-site mentoring. Following are examples of implementation work from the 3 cohorts.

\section{Cohort 1: Washington University in St. Louis}

After an assessment of barriers and facilitators, consistent with the use of evaluative and iterative strategies as an implementation method, ${ }^{9}$ we developed and implemented the Electronic Health Record (EHR)-enabled Evidence-based Smoking Cessation Treatment (ELEVATE) program $^{10,11}$ that enacted a paradigm shift to a point-ofcare tobacco treatment model. The ELEVATE program is a bundled implementation strategy that includes (1) an EHR-enabled team care workflow that provides scripted advice and an opt-out offer of counseling via phone, text, or app by closed-loop referrals to national quit-lines and smokefree.gov (reflecting a change to the infrastructure to facilitate implementation ${ }^{9}$ ), and (2) a learning health system approach (eg, a cycle of data-to-knowledge, knowledge-to-practice, and practice-to-data, including feedback). ELEVATE increased reach, defined as the proportion of current smokers who received evidence-based tobacco treatment (from $3.2 \%$ among 815 patients who smoke to $32.8 \%$ among 2,511 patients who smoke) (see Figure 1), and yielded a smoking cessation rate of $29.5 \%$ at 6 months. ${ }^{12}$ The program cost at $\$ 70 /$ patient was much lower than most tobacco treatment programs. ${ }^{8}$ Consequently, ELEVATE has been scaled up from 21 to more than 900 clinics at Washington University/BJC HealthCare, and serves as a model for a sustainable C3I program.

\section{Cohort 2: Mayo Clinic Cancer Center}

The Mayo Clinic Cancer Center (MCCC) used a change to its infrastructure ${ }^{9}$ to facilitate implementation by using an "opt-out" clinical workflow that systematically screened and referred all tobacco-using patients to a Nicotine Dependence Center (NDC). ${ }^{13}$ Through a phased implementation process spanning across Minnesota, Wisconsin, Arizona, and Florida, the workflow was implemented across ambulatory practices. From July 2019 to May 2021, 3,727 NDC referrals were received. As of April 2021, 681 (18\%) of those referred had completed $\geq 1$ NDC appointment, of which 173 (27\%) were relisted as former smokers in the EHR. C3I support also catalyzed the implementation of changes to infrastructure at the health system level by including structured documentation in the EHR to record e-cigarette use, ${ }^{14}$ the establishment of a tobacco and e-cigarette registry, an EHR alert for scheduling staff to update tobacco use history, a patient engagement video delivered via an appointment portal, and use of the NCI's Cancer Patient Tobacco Use

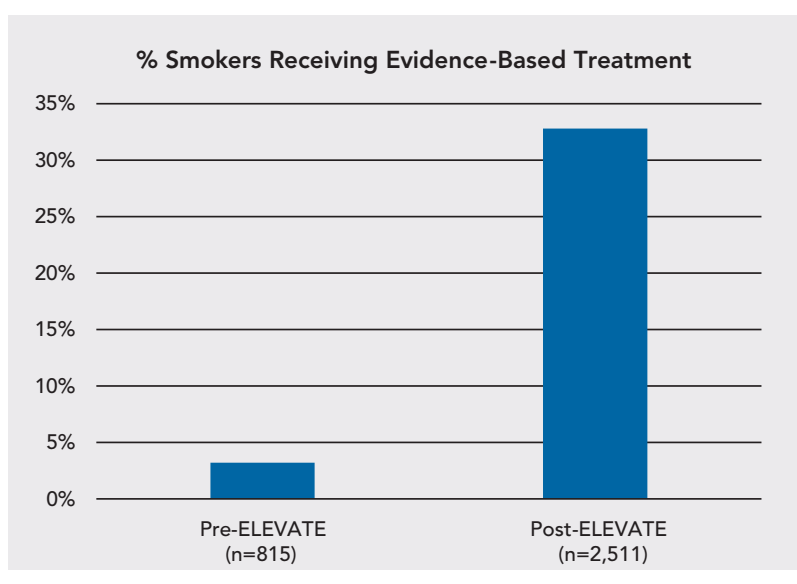

Figure 1. Illustrative example of impact from C3I-supported efforts on reach from Washington University in St. Louis.

Note: Relative risk (adjusted for age, sex, race), 10.06 (95\% Cl, 6.89-14.68); $P<.0001$ in generalized estimating equation models.

Questionnaire. Changing the health system infrastructure as an implementation strategy using an "opt-out" referral workflow that does not require clinician involvement demonstrated effectiveness and sustainability in oncology practices.

\section{Cohort 3: City of Hope Cancer Center - Beckman Research Institute}

The City of Hope Cancer Center - Beckman Research Institute $(\mathrm{COH})$ conducted a needs assessment and surveyed cancer center staff attitudes regarding tobacco treatment, consistent with using evaluative and iterative implementation strategies. ${ }^{15}$ The tobacco use rate $(97.9 \%$ of patients assessed) was $4.0 \%$ to $9.7 \%$ of oncology patients across 36 academic and community sites. ${ }^{15} \mathrm{COH}$ increased resource commitment to the $\mathrm{COH}$ cessation program, and senior leaders initiated monthly "Moonshot Shoutout" communications to staff to promote the establishment of a tobacco treatment program, consistent with training and educating stakeholders as an implementation strategy. ${ }^{15}$ The multidisciplinary cessation program developed and distributed culturally sensitive and multilingual educational materials to patients. Training modules were built for smoking cessation champions and clinical staff to develop stakeholder interrelationships. ${ }^{15}$ To support efficient and effective access to tobacco treatments, a clinical informatics team modified the EHR to include Best Practice Advisories, initially with an "opt-in" approach but transitioning to an automated "opt-out" referral to cessation treatment (changed infrastructure) and the use of "SmartPhrases" (abbreviations or a few words to pull long phrases or sentences into patient's EHR). An in-person cessation clinic was supplemented by telehealth, online, and quit-line counseling. To overcome multidepartmental "silos" and geographic barriers, $\mathrm{COH}$ 


\section{Table 1. Immediate and Long-Term Goals of the C3I Implementation Science Working Group}

\begin{tabular}{|c|c|}
\hline Immediate Goals & Long-Term Goals \\
\hline Survey working group membership to refine goals & $\begin{array}{l}\text { Publish a review paper on implementation science related to tobacco use } \\
\text { treatment and cancer }\end{array}$ \\
\hline $\begin{array}{l}\text { Build a community of people interested in implementation science related } \\
\text { to tobacco use treatment and cancer }\end{array}$ & $\begin{array}{l}\text { Develop a cross-institutional consultation and mentoring service applying } \\
\text { implementation science to tobacco use treatment for cancer centers }\end{array}$ \\
\hline Develop a resource list of implementation science publications & $\begin{array}{l}\text { Develop a toolkit for applying implementation science to tobacco use } \\
\text { treatment for cancer centers }\end{array}$ \\
\hline Explore avenues for bridging activities across $\mathrm{C} 31$ working groups & $\begin{array}{l}\text { Increase the number of multi-institutional collaborative grants and } \\
\text { publications on implementation science related to tobacco use treatment } \\
\text { and cancer }\end{array}$ \\
\hline $\begin{array}{l}\text { Encourage graduate students and research fellows to pursue } \\
\text { implementation science-related research relevant to tobacco use } \\
\text { treatment and cancer }\end{array}$ & $\begin{array}{l}\text { Increase the number of student/research fellow led publications on } \\
\text { implementation science related to tobacco use treatment and cancer }\end{array}$ \\
\hline $\begin{array}{l}\text { Conduct focused assessment to understand barriers and facilitators of } \\
\text { implementing tobacco use treatment programs in community oncology } \\
\text { settings }\end{array}$ & $\begin{array}{l}\text { Promote the sustainability of tobacco use treatment in community } \\
\text { oncology care settings using implementation science tools }\end{array}$ \\
\hline
\end{tabular}

leadership designated multilevel cessation champions (physicians, advance practice providers, and oncology nurses) at each academic and community site. For evaluation and monitoring, $\mathrm{COH}$ is tracking referrals, cessation clinic engagement, and smoking abstinence, as well as staff attitudes, as a form of audit and feedback as an implementation strategy. ${ }^{9}$ Because $\mathrm{COH}$ is in the third cohort of the C3I initiative, data collection to assess impact is currently ongoing.

\section{Implementation Science Working Group Agenda}

The C3I Implementation Science Working Group, co-led by Drs. Chen, Ramsey, and Schnoll (each with roles in C3I and ISC3), began in December 2020 to bring together clinicians, scientists, and policymakers across C3I sites who share the common interest in implementation science and treating tobacco use in the oncologic context. Through monthly meetings, smaller interest groups, and activities, this working group will help strengthen the national community committed to implementation science and tobacco use in the cancer context, and engage in manuscripts, cross-institutional research and mentoring consultation, and toolkit development to enhance the state of the science in this area, including the cataloguing of effective implementation strategies that promote treatment engagement, lead to reduced tobacco use rates, and facilitate sustainability, similar to past efforts. ${ }^{9}$

The working group began with a member survey of interests in implementation science domains (eg, reach, sustainability), priorities, and perceptions of barriers and facilitators to program sustainability. Among 13 respondents representing 12 cancer centers, program effectiveness (85\%), implementation strategies (70\%), and context (70\%) were the top 3 interests. Collaborating on papers and developing a toolkit of effective implementation strategies for other groups interested in implementing tobacco cessation treatment services within the oncologic context were considered top priorities. Funding availability was cited as the most frequent barrier to sustaining programs (58\%), and modifications to the EHR was cited as the most feasible method to sustaining programs (36\%). These data helped form the mission and goals of the working group.

Based on initial meetings and data collection, the working group members established a set of immediate and long-term goals (Table 1). In the near term, the working group hopes to stimulate interest in implementation science and tobacco treatment, broaden the community of people interested in implementation science and tobacco treatment, and explore avenues for bridging across C3I working groups. In the long-term, the working group hopes to facilitate multiple papers focused on implementation science and tobacco treatment, develop a cross-institutional consulting service for implementation science and tobacco treatment (including with cancer treatment facilities not supported previously by the C3I initiative), devise a toolkit of implementation strategies for other cancer centers (within and beyond C3I) to use to establish a sustainable tobacco cessation program, and increase the number of multi-institutional grants and publications focused on this area. To date, we have established writing teams (supported by the co- chairs) who are working on 3 papers: (1) the present paper describing the working group, (2) a state-of- the-science paper describing the current literature and gaps and priorities for the field, and (3) a data-based paper characterizing implementation strategies used by C3I programs and evaluating their effects on program reach and effectiveness. The working group has also facilitated new consultations between C3I sites on program implementation and supported efforts to develop novel ideas for future implementation science and tobacco control research. 


\section{Conclusions}

The C3I Implementation Science Working Group facilitates and promotes the overall goals of the C3I Moonshot program, including the provision of direct support to initiative members on the implementation of sustainable efforts to ensure that patients with cancer are screened for tobacco use and provided with evidence-based tobacco treatments. The case studies presented here illustrate the use of implementation strategies (eg, changed infrastructure) to achieve these goals, which can be adopted by other C3I sites or other cancer treatment facilities across the nation not formally involved in C3I. By building an implementation science community dedicated to addressing the gap in tobacco treatment utilization across NCI-designated Cancer Centers, we aim to advance C3I's mission to mitigate the adverse impacts of tobacco use on cancer risk and outcomes.

\section{Cancer Center Cessation Initiative Implementation Science Working Group Members}

Robert Schnoll, PhD ${ }^{1, *}$; Li-Shiun Chen, MD, MPH, ScD 2,*; Alex T. Ramsey, $\mathrm{PhD}^{2, *}$; Cary A. Presant, MD, FACP, FASCO ${ }^{3, \dagger}$; Thulasee Jose, MD ${ }^{4,+}$; Kimlin T. Ashing, $\mathrm{PhD}^{5,+}$; Donna Shelley, MD, MPH ${ }^{6,+}$; Steven L. Bernstein, $\mathrm{MD}^{7,+}$; Linda Fleisher, $\mathrm{PhD}, \mathrm{MPH}^{8, \dagger}$; Ramzi G. Salloum, $\mathrm{PhD}^{9,+}$; Graham W. Warren, $\mathrm{MD}, \mathrm{PhD}^{10,+}$; Kara P. Wiseman, PhD, MPH ${ }^{11,+}$; Janice A. Blalock, PhD ${ }^{12, \neq^{\prime}}$; Neely A. Dahl, MPH ${ }^{13, \pm}$; Edlira B. Farka, APRN ${ }^{14, \pm}$; Donna Manders, MPH, CTTS $^{15, \neq}$; Jamie S. Ostroff, PhD ${ }^{16, \neq}$; Elyse R. Park, PhD, MPH 17, ; Robert Adsit, MEd ${ }^{18, \S ;}$; Sarah D. Hohl, MPH, PhD ${ }^{19, \$}$; Mara Minion, MA ${ }^{19, \$ ;}$ Danielle Pauk, $\mathrm{BS}^{19, \S}$; and Betsy Rolland, PhD, MLIS, MPH ${ }^{19,20, \S}$

1Department of Psychiatry and Abramson Cancer Center, University of Pennsylvania, Philadelphia, Pennsylvania; ${ }^{2}$ Department of Psychiatry and Siteman Cancer Center, Washington University in St. Louis, St. Louis, Missouri; ${ }^{3}$ Department of Medical Oncology and Therapeutics Research, City of Hope
Comprehensive Cancer Center, and Beckman Research Institute, Duarte, California; ${ }^{4}$ Department of Anesthesiology and Perioperative Medicine, Mayo Clinic, Rochester, Minnesota; ${ }^{5}$ Department of Population Sciences, City of Hope National Medical Center, Duarte, California; ${ }^{6}$ Department of Policy and Public Health Management, School of Global Public Health, New York University, New York, New York; ${ }^{7}$ Department of Emergency Medicine, Geisel School of Medicine, Dartmouth College, Hanover, New Hampshire; ${ }^{8}$ Fox Chase Cancer Center, Philadelphia, Pennsylvania; ${ }^{9}$ Department of Health Outcomes \& Biomedical Informatics, College of Medicine, University of Florida, Gainesville, Florida; ${ }^{10}$ Department of Radiation Oncology, Department of Cell and Molecular Pharmacology, Medical University of South Carolina, Charleston, South Carolina; ${ }^{11}$ Department of Public Health Sciences, School of Medicine, University of Virginia, Charlottesville, Virginia; ${ }^{12}$ Department of Behavioral Science, University of Texas MD Anderson Cancer Center, Houston, Texas; ${ }^{13}$ University of Virginia Cancer Center, Charlottesville, Virginia; ${ }^{14}$ Huntsman Cancer Institute, University of Utah, Salt Lake City, Utah; ${ }^{15}$ Seattle Cancer Care Alliance, Seattle, Washington; ${ }^{16}$ Department of Psychiatry and Behavioral Sciences, Memorial Sloan Kettering Cancer Center, New York, New York; ${ }^{17}$ Department of Psychiatry, Massachusetts General Hospital (MGH), Boston, Massachusetts; ${ }^{18}$ Department of Medicine, Center for Tobacco Research and Intervention, School of Medicine and Public Health University of Wisconsin-Madison, Madison, Wisconsin; ${ }^{19}$ Carbone Cancer Center, University of Wisconsin-Madison, Madison, Wisconsin; and ${ }^{20}$ Institute for Clinical and Translational Research, University of Wisconsin-Madison, Madison, Wisconsin.

"Working Group Chair; ${ }^{+}$Writing Group Author; ${ }^{\ddagger}$ Working Group Author; ${ }^{\S}$ Coordinating Center Author.

Submitted July 26, 2021; final revision received September 14, 2021 accepted for publication September 16, 2021.

Disclosures: G.W. Warren has disclosed participating in research for the Agency for Healthcare Research and Quality and serving as a consultant for the Canadian Partnership Against Cancer. The remaining individuals have disclosed no relevant financial relationships.

Funding: This supplement was funded by the $\mathrm{C} 3 \mathrm{I}$ Coordinating Center contract from the National Cancer Institute (CRDF Award \#66590). In addition, authors received funding for their $\mathrm{C} 3 \mathrm{I}$ participation via a supplement to their $\mathrm{NCl}$ P30 cancer center support grant during the period 2017-2021.

Correspondence: Thulasee Jose, MD, Department of Anesthesiology and Perioperative Medicine, Mayo Clinic, 200 First Street SW, Rochester, MN 55905. Email: thulasee.jose@bhset.edu

\section{References}

1. Warren GW, Evans WK, Dresler C. Critical determinants of cancer treatment outcomes: smoking must be addressed at the highest levels in cancer care. J Thorac Oncol 2021;16:891-893.

2. Croyle R, Morgan G, Fiore M. Addressing a core gap in cancer care: the $\mathrm{NCl}$ Cancer MoonshotSM initiative to help oncology patients stop smoking. N Engl J Med 2019;380:512.

3. Patnode CD, Henderson JT, Coppola EL, et al. Interventions for tobacco cessation in adults, including pregnant persons: updated evidence report and systematic review for the US Preventive Services Task Force. JAMA 2021;325:280-298.

4. Warren GW, Marshall JR, Cummings KM, et al. Addressing tobacco use in patients with cancer: a survey of American Society of Clinical Oncology members. J Oncol Pract 2013;9:258-262.

5. Ramaswamy AT, Toll BA, Chagpar AB, et al. Smoking, cessation, and cessation counseling in patients with cancer: a population-based analysis. Cancer 2016;122:1247-1253.

6. D'Angelo H, Rolland B, Adsit R, etal. Tobacco treatment program implementation at $\mathrm{NCl}$ cancer centers: progress of the $\mathrm{NCl}$ Cancer Moonshot-funded Cancer Center Cessation Initiative. CancerPrev Res (Phila) 2019;12:735-740.

7. D'Angelo H, Ramsey AT, Rolland B, et al. Pragmatic application of the RE-AIM framework to evaluate the implementation of tobacco cessation programs within $\mathrm{NCl}$-designated cancer centers. Front Public Health 2020;8:221.

8. Salloum RG, D'Angelo H, Theis RP, et al. Mixed-methods economic evaluation of the implementation of tobacco treatment programs in National
Cancer Institute-designated cancer centers. Implement Sci Commun 2021:2:41

9. Powell BJ, Waltz TJ, Chinman MJ, et al. A refined compilation of implementation strategies: results from the Expert Recommendations for Implementing Change (ERIC) project. Implement Sci 2015;10:21.

10. Ramsey AT, Chiu A, Baker T, et al. Care-paradigm shift promoting smoking cessation treatment among cancer center patients via a low-burden strategy, Electronic Health Record-Enabled Evidence-Based Smoking Cessation Treatment. Transl Behav Med 2020;10:1504-1514.

11. Ramsey AT, Baker TB, Pham G, et al. Low burden strategies are needed to reduce smoking in rural healthcare settings: a lesson from cancer clinics. Int J Environ Res Public Health 2020;17:1728

12. Craig EJ, Ramsey AT, Baker TB, et al. Point of care tobacco treatment sustains during COVID-19, a global pandemic [published online August 18, 2021]. Cancer Epidemiol, doi: 10.1016/j.canep.2021.102005

13. Jose T, Ohde JW, Hays JT, et al. Design and pilot implementation of an electronic health record-based system to automatically refer cancer patients to tobacco use treatment. Int J Environ Res Public Health 2020; 17:4054.

14. Jose T, Hays JT, Warner DO. Improved documentation of electronic cigarette use in an electronic health record. Int J Environ Res Public Health 2020;17:E5908.

15. Presant CA, Salgia R, Kulkarni $P$, et al. Implementing lung cancer screening and prevention in academic centers, affiliated network offices and collaborating care sites. J Clin Med 2020;9:1820. 